TARGETED THERAPY

\title{
Ripretinib turns off the switch in GIST
}

More than $85 \%$ of cases of gastrointestinal stromal tumour (GIST) harbour mutations in the genes encoding the receptor tyrosine kinases KIT and platelet-derived growth factor receptor a (PDGFRa). Treatment of metastatic GIST has been transformed by kinase inhibitors such as imatinib, which is considered to be one of the most successful targeted agents ever developed. However, this type of inhibitor only blocks a limited number of KIT mutants, and secondary resistance mutations eventually emerge. Now, Daniel Flynn, from Deciphera Pharmaceuticals, and collaborators have designed ripretinib, an investigational tyrosine kinase inhibitor that targets a broad spectrum of KIT and PDGFRa mutants.

"Kinases are known to have embedded switches that regulate protein conformation and enzymatic activity", explains Flynn. KIT and PDGFRa have two switches: an inhibitory switch in the intracellular juxtamembrane domain (JMD, which is encoded by exon 11 in KIT and exon 12 in PDGFRA) and an activation switch in the kinase domain (which is encoded by exons 17 and 18 in KIT and exons 18 and 19 in PDGFRA). Phosphorylation of one or more switch amino acids changes the conformation from type I (active) to type II (inactive) and vice versa, turning the kinases 'off' and 'on'. Most mutations in GIST (found in approximately $70 \%$ of the patients) are loss-of-function mutations in the inhibitory switch, and lead to a shift in conformation towards a type I active form. However, a small percentage of primary activating mutations and almost all secondary resistance mutations in GIST are gain-of-function mutations in the activating switch that stabilize the active type I conformation and lead to an even more uncontrolled activation of KIT, says Flynn. These mutations confer resistance to FDA-approved type II kinase inhibitors such as imatinib, regorafenib and sunitinib.

To develop a novel KIT and

PDGFRa inhibitor that is designed to inhibit activating mutations in all relevant known exons, including in the activation loop, Flynn and colleagues used structure-based drug design to create an inhibitor that could bind to key amino acid residues within the KIT switching mechanism.

In vitro, ripretinib and other analogues both prevented KIT from adopting a type I active conformation and locked it in the inactive conformation. The authors showed that ripretinib inhibited mutations across all six exons known to be mutated in KIT-driven GIST as well as mutations in PDGFRa in a panel of GIST cell lines and cells expressing primary and drug-resistant KIT or PDGFRa mutants. Ripretinib also inhibited proliferation of KIT and PDGFRa mutant cell lines derived from GIST, systemic mastocytosis, leukaemia and lung cancer. "Furthermore, saturation mutagenesis studies revealed that ripretinib was able to withstand emergence of de novo KIT resistance mutations", says Flynn.

In vivo, treatment of mice carrying mutant KIT GIST cell line xenografts with two doses of 25 and $100 \mathrm{mg} / \mathrm{kg} /$ day of ripretinib resulted in significant tumour regression. At the high dose, out of 10 mice, 6 showed complete and 4 partial tumour regression. Both doses were well tolerated and although tumours relapsed after the end of the dosing period, survival at day 68 was $100 \%$ for ripretinib-treated mice compared with $25 \%$ for vehicle-treated mice.

In an imatinib-resistant GIST patient-derived xenograft model, repeated dosing of $50 \mathrm{mg} / \mathrm{kg}$ twice daily for 28 days resulted in tumour regression and extended survival (100\% compared with $10 \%$ for vehicle-treated mice at day 57).

Finally, on the basis of the promising preclinical data the authors

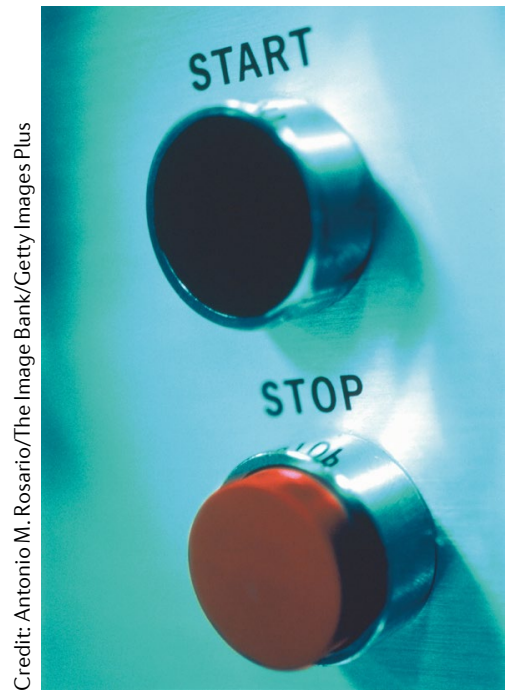

conducted a first-in-human study to evaluate the safety and tolerability of ripretinib (NCT02571036). Patients with drug-resistant GIST harbouring a broad spectrum of KIT mutations were enrolled during the dose-escalation phase of study. At first assessment, two representative patients in this phase I study showed reduction of KIT mutant allele frequency in circulating tumour DNA.

Ripretinib is currently being studied in two phase III trials: INVICTUS and INTRIGUE in patients with GIST. The results from these trials could confirm the potential of this investigational inhibitor. Furthermore, the switch control inhibition approach to kinases could have broader applicability. "We have extended this proprietary kinase switch control inhibitor platform to develop highly selective, potent small-molecule drug candidates that have the potential to directly inhibit activation of other kinases", says Flynn. These compounds include inhibitors of angiopoietin-1 receptor TIE2 and macrophage colony-stimulating factor 1 receptor (CSF1R), which are both implicated in tumour progression.

M. Teresa Villanueva, Senior Editor Nature Reviews Drug Discovery This article is modified from the original in Nat. Rev. Drug Discov. (https://doi.org/10.1038/d41573-019-00099-4). de novo KIT resistance mutations

ORIGINAL ARTICLE Smith, B. D. et al. Ripretinib (DCC-2618) is a switch control kinase inhibitor of a broad spectrum of oncogenic and drug-resistant KIT and PDGFRA variants. Cancer Cell 35, 738-751 (2019) 